usw.), wobei der Prozentsatz von Kernen mit dieser Besonderheit mit zunehmendem Polyploidiegrad steigt. In seltenen Fällen finden sich auch Sonderungen nach Aneuploidzahlen, wobei die von den aneuploiden Kernen her bekannten Zahlen 12, 27, 33 und 54 eine besondere Rolle spielen ${ }^{\mathbf{1}}$ ).

Tabelle 1. Die aneuploiden Chromosomenzahlen in der unbehandelten hepatektomierten Leber 6 Monate alter Ratten $(2 n=42)$

Gefundene Chromosomenzahl (Chr. Z.) und Zellenzahl (Zell. Z.)

\begin{tabular}{l|lll|l|l|lllllll|l|l|} 
Chr. Z. & 12 & 13 & 16 & 27 & 33 & 35 & 36 & 37 & 44 & 48 & 49 & 50 & 54 & 57 \\
\hline
\end{tabular} \begin{tabular}{l|lll|l|l|lllllll|l|l}
\hline Zell. Z. & 3 & 1 & 1 & 28 & 47 & 1 & 3 & 1 & 1 & 1 & 1 & 4 & 36 & 1
\end{tabular}

\begin{tabular}{l|lllll|l|llllllll} 
Chr. Z. & 58 & 60 & 67 & 69 & 74 & 75 & 78 & 79 & 90 & 96 & 98 & 99 & 117 & 138
\end{tabular} \begin{tabular}{l|lllll|l|llllllll}
\hline Zell. Z. & 1 & 1 & 1 & 6 & 1 & 17 & 1 & 1 & 3 & 2 & 1 & 1 & 2 & 1
\end{tabular}

Bei den aneuploiden Metaphasen finden wir die Genomsonderung stärker als bei den euploiden Kernen ausgeprägt, und zwar wird stets nach einem oder mehreren ganzen Genomen und nach einer aneuploiden Restgruppe gesondert, die infolge der Bevorzugung bestimmter Chromosomenzahlen meist aus $6,12,27,33$ oder 54 Chromosomen besteht, also wieder aus den bevorzugt vorhandenen Aneuploidzahlen.

Die verhältnismäßig wenigen auftretenden aneuploiden Stufen in der Rattenleber dürften also dadurch zustande kommen, daß in einem Zellkern mit Genomsonderung durch eine multipolare Spindel die aneuploide Chromosomengruppe und der Rest des Chromosomensatzes an zwei auf ein und derselben Spindelseite getrennt nebeneinander ausgebildete Spindelpole transportiert werden und sich dort zwei selbständige Tochterkerne statt nur eines bilden. Derselbe Effekt könnte auch durch eine Amitose erreicht werden, welche die beiden gesonderten Chromosomengruppen voneinander trennt. Welche aneuploiden Chromosomenzahlen und wieviele aneuploide Kerne also in einem Gewebe auftreten, ist in erster Linie von dem Umfang und der Art und Weise einer Genomsonderung bestimmt.

Mit Unterstützung der Deutschen Forschungsgemeinschaft.

Forstbotanisches Institut der Universität, Freiburg $i, B r$.

EBERHARD GLÄSs

Eingegangen am 30. Oktober 1957

1) GLÄss, E.: Chromosoma 7, 655 (1956); 8, 468 (1957). 9) MARQuardt, H., u. E. GLÄss: Chromosoma 8, 617 (1957).

\section{Die Veränderungen der Häufigkeit euploider und aneuploider Chromosomenzahlen in der hepatektomierten Rattenleber bei Buttergelb-Verfütterung}

An Ratten, die von Tieren aus den Zuchten Druckreys abstammten, wurde ein typischer Durchfütterungsversuch mit Buttergelb durchgeführt ( $5 \mathrm{mg}$ Dimethylamino-azobenzol täglich dem Futter zugefügt, jedoch im Gegensatz żu DRUCKREY ${ }^{1}$ ) nicht zurückgewogen) und 48 Std nach Hepatektomie die Leber von Tieren fixiert, die $220,320,400,640,790$ und $1020 \mathrm{mg}$ Buttergelb erhalten hatten und noch keine Geschwulst

Tabelle 1. Die Häufigkeit euploider und aneuploider Chromosomenzahlen in der Rattenleber bei steigender Buttergelbdosis

\begin{tabular}{|c|c|c|c|c|c|c|c|}
\hline Dosis & 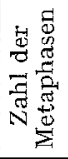 & $\begin{array}{l}\overrightarrow{\mathrm{N}} \\
\mathrm{l} \\
\stackrel{\mathrm{H}}{\mathrm{B}}\end{array}$ & 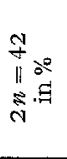 & $\begin{array}{l}\text { o } \\
\text { il } \\
\text { s. } \\
\text { m }\end{array}$ & 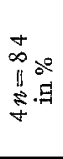 & $\begin{array}{l}\frac{2}{2} \\
\frac{1}{1} .9 \\
\text { m }\end{array}$ & 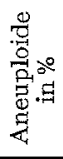 \\
\hline Unbehandelt. & 328 & 8,5 & 43,5 & 9,4 & 20,2 & 6,2 & 12,2 \\
\hline 400 und $640 \mathrm{mg}$ & 769 & 6,2 & 26,7 & 12,8 & 9,1 & 6,1 & 37,1 \\
\hline 790 und $1020 \mathrm{mg}$ & 722 & 6,8 & 13,3 & 10,5 & 9,1 & 5,7 & 54,6 \\
\hline
\end{tabular}

besaßen. Gegenüber den normalen Chromosomenverhältnissen treten mit steigender Buttergelbdosis einschneidende Veränderungen in der Häufigkeit sowohl der euploiden wie der aneuploiden Chromosomenzahlen auf. Vor allen Dingen nimmt die Zahl der normal-diploiden Mitosen von $43 \%$ auf $13 \% \mathrm{ab}$, während die Zahl der aneuploiden Metaphasen von $12 \%$ auf $55 \%$ ansteigt; die übrigen euploiden Stufen reagieren schwächer oder gar nicht (Tabelle 1).

Unter den aneuploiden Stufen nehmen diejenigen der „,bevorzugten Chromosomenzahlen" 27, 33 und 54 (vgl. die vorhergehende Originalmitteilung) $a b$, während neue, aneuploide Zahlen auftreten, so daß die Zahl der nicht bevorzugten Klassen von 10 auf 33 steigt und außerdem als neue bevorzugte Zahlen 36,48 und 96 auftreten, von denen die Klasse mit 48 Chromosomen von $0,8 \%$ auf $14,7 \%$ zunimmt (Tabelle 2). Die erste bei $220 \mathrm{mg}$ Buttergelb bereits feststellbare Anderung besteht in einer starken Zunahme der Metaphasen mit Genomsonderung.

Tabelle 2. Die bevorzugten aneuploiden Chromosomenzahlen in der Rattenleber bei steigender Buttergelbdosis

\begin{tabular}{|c|c|c|c|c|c|c|c|c|c|c|}
\hline Dosis & 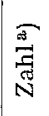 & $\begin{array}{l}27 \\
\text { in } \\
\%\end{array}$ & $\begin{array}{l}33 \\
\text { in } \\
\%\end{array}$ & $\begin{array}{l}36 \\
\text { in } \\
\%\end{array}$ & $\begin{array}{l}48 \\
\text { in } \\
\%\end{array}$ & $\begin{array}{l}54 \\
\text { in } \\
\%\end{array}$ & $\begin{array}{l}69 \\
\text { in } \\
\%\end{array}$ & $\begin{array}{l}75 \\
\text { in } \\
\%\end{array}$ & $\begin{array}{l}96 \\
\text { in } \\
\%\end{array}$ & 茎 \\
\hline $\begin{array}{l}\text { Unbehandelt. } \\
400 \text { und } 640 \mathrm{mg}\end{array}$ & $\begin{array}{l}129 \\
285\end{array}$ & $\begin{array}{r}17,8 \\
8,8\end{array}$ & $\begin{array}{l}30,2 \\
15,1\end{array}$ & $\begin{array}{l}2,3 \\
2,8\end{array}$ & $\begin{array}{r}0,8 \\
12,2\end{array}$ & $\begin{array}{l}24,0 \\
19,7\end{array}$ & $\begin{array}{l}3,9 \\
3,8\end{array}$ & $\begin{array}{r}8,5 \\
11,2\end{array}$ & $\begin{array}{l}0,8 \\
4,9\end{array}$ & $\begin{array}{l}10 \\
22\end{array}$ \\
\hline 790 und $1020 \mathrm{mg}$ & 394 & 5,3 & 8,1 & 5,8 & 14,7 & 14,9 & 7,3 & 9,9 & 4,3 & 33 \\
\hline
\end{tabular}

a) Zahl der Metaphasen. - b) Anzahl nicht bevorzugter Klassen.

Da die hier nur kurz dargestellten Befunde an hepatektomierten Lebern erhoben wurden, lassen sich folgende Schlüsse ziehen: 1. Unter dem Einfluß von Buttergelb vollziehen sich Veränderungen, durch welche die normalen diploiden Kerne zum großen Teil am Eintritt in Regenerationsmitosen gehindert werden. 2. Vorbereitet durch eine $\mathrm{Zu}$ nahme der Kerne mit Genomsonderung, wird ein Ablauf in Gang gebracht, durch den die Zahl der aneuploiden Zellen stark erhöht wird; dabei entstehen Zellen mit bestimmten Chromosomenzahlen, die eine gegenüber den Normal-Kernklassen erhöhte Teilungsfähigkeit haben. 3. Mehrere, neu aufgetretene, aneuploide Kernklassen besitzen die erhöhte Teilungsfähigkeit.

Gestuitzt werden diese Schlußfolgerungen durch neuere Erfahrungen an Einzelzellkulturen von Affen-Nierenepithel, in denen nach einer bestimmten Kulturzeit aneuploide Zellen entstehen, die ähnlich wie in unserem Fall ganz bestimmte Chromosomenzahlen zwischen den euploiden Stufen besitzen, die sich in ihren morphologischen und physiologischen Eigenschaften in Richtung auf Malignität von den Ausgangszellen unterscheiden und die eine erhöhte Teilungsfähigkeit selbst in einem Medium haben, das für die Normalzellen ungeeignet ist ${ }^{2}$. Ähnliches wurde auch an menschlichen Gewebezellen in Kultur nachgewiesen $\left.\left.{ }^{3}\right),{ }^{4}\right),{ }^{5}$ ).

$\mathrm{Da}$ angenommen werden $\mathrm{mu} \beta$, daß die bei der Leberregeneration unter hohen Buttergelbdosen noch teilungsfähigen Kernklassen im wesentlichen auch am späteren Aufbau des cancerösen Zellmaterials beteiligt sein werden, kann mit der Möglichkeit gerechnet werden, daß bei der Cancerisierung durch Buttergelb zunächst eine Phase vorhanden ist, bei der das Spektrum aneuploider Zellen verbreitert wird. Dabei sind nur Kerne mit bestimmten aneuploiden Chromosomenzahlen so weit teilungsfähig, daß sie in der Mitose erfaßt werden. Gleichzeitig verändern sich die Bedingungen in der Leber derartig, daß auf der einen Seite die diploiden Zellen am Eintritt in die Mitose gehindert werden, auf der anderen Seite aneuploide Kerne mit bestimmten Chromosomenzahlen eine erhöhte Teilungsbereitschaft entwickeln. Sofern nach oder mit dieser Eigenschaft weitere hinzukommen, die für eine krebsig entartete Zelle typisch sind, werden dann die auf diese Weise herausselektionierten und ständig bösartiger werdenden Kern- und Zelltypen den Tumor aufbanen. Experimente, diese Hypothese zu prüfen, befinden sich in Gang.

Mit Unterstützung der Deutschen Forschungsgemeinschaft.

Herrn Prof. Druckrey, Freiburg i. Br., danken wir herzlich für die Bereitstellung der Tiere und seinen Rat bei der Versuchsdurchführung, Herrn Dr. KERCH, Bern, für die Hilfe bei der Beschaffung der neuesten Literatur.

Forstbotanisches Institut der Universität, Freiburg $i$. Br. Hans Marquardt und EBerhard GLäss

Eingegangen am 30. Oktober 1957

1) Druckrey, H., u. K. KupfMüller: Z. Naturforsch. 36, 254 (1948). - - 2) PArker, R. C.: Vortr. 4. Internat. Poliomyelitis Conference Genf. Excerpta med. 1957, 81. - ${ }^{3}$ ) Moore, A. E.: Science [Lancaster, Pa.] 124, 127 (1956). - Vortr. 4. Internat. Poliomyelitis Conference Genf. Excerpta med. 1957, 81. - 4) Puck, Tr. T.: Vortr. 4. Internat. Poliomyelitis Conference Genf. Excerpta med. 1957, 80. - ${ }^{5}$ ) LevaN, A.: Cancer [N. Y.] 9, 648 (1956). 